\title{
Seronegative Wegener's Granulomatosis Presenting as a Multiple Cranial Neuropathy
}

Sara Machado ${ }^{1,2 *}$, Nuno Inácio ${ }^{1}$, Filipe Paulas ${ }^{3}$, Luísa Biscoito ${ }^{4}$, João Paulo Farias ${ }^{5}$, Marta Amaral ${ }^{3}$, José Alves ${ }^{3}$ and Amélia Nogueira Pinto ${ }^{2}$

${ }^{1}$ Department of Neurology, Hospital Professor Doutor Fernando Fonseca, EPE, Amadora, Portugal

${ }^{2}$ Institute of Neurology, UCL, National Hospital for Neurology and Neurosurgery, London, UK

3IV Department of Internal Medicine, Hospital Professor Doutor Fernando Fonseca, EPE, Amadora, Portugal

${ }^{4}$ Department of Neuroradiology, Hospital de Santa Maria, Centro Hospitalar de Lisboa Norte EPE, Lisboa, Portugal

${ }^{5}$ Department of Neurosurgery, Hospital de Santa Maria, Centro Hospitalar de Lisboa Norte EPE, Amadora, Portugal

\begin{abstract}
Wegener's granulomatosis (WG) is an immune-mediated systemic vasculitis of unknown etiology that can be seen in almost any system. It classically affects the upper and lower airways, lungs and kidneys. Despite its possibility of generalization, neurological involvement is hardly seen. When it occurs, WG tends to affect the peripheral nervous system, often as sensory-motor polyneuropathy or mononeuritis multiplex. Involvement of cranial nerves is much less frequent, and the most affected is the optic nerve. Involvement of the central nervous system is even rarer but there are reports including cerebrovascular events, seizures, cerebritis, diabetes insipidus and pachymeningitis.

In 1990, four criteria have been identified for the clinical diagnosis of WG by the American College of Rheumatology, which are still in effect and include upper airways inflammation, pulmonary nodules and microhematuria. A patient who meets at least 2 of the criteria may be diagnosed as having classical WG with a sensitivity of $88 \%$ and a specificity of $92 \%$. Usually there is positivity for antineutrophil cytoplasmatic antigene (ANCA) but its absence does not exclude this diagnosis, especially when the clinical picture is highly suggestive. We present a case of palsy of the lower cranial nerves as the initial presentation of a seronegative WG.
\end{abstract}

Keywords: Wegener's granulomatosis; Neurological involvement; Cranial neuropathy; Aseptic meningitis

\section{Summary}

Wegener's granulomatosis (WG) is an immune-mediated systemic vasculitis of unknown etiology that can be seen in almost any system. It classically affects the upper and lower airways, lungs and kidneys. Despite its possibility of generalization, neurological involvement is hardly seen. When it occurs, WG tends to affect the peripheral nervous system, often as sensory-motor polyneuropathy or mononeuritis multiplex [1]. Involvement of cranial nerves is much less frequent, and the most affected is the optic nerve [1]. Involvement of the central nervous system is even rarer but there are reports including cerebrovascular events, seizures, cerebritis, diabetes insipidus and pachymeningitis [2].

In 1990, four criteria have been identified for the clinical diagnosis of WG by the American College of Rheumatology [3], which are still in effect and include upper airways inflammation, pulmonary nodules and microhematuria. A patient who meets at least 2 of the criteria may be diagnosed as having classical WG with a sensitivity of $88 \%$ and a specificity of $92 \%$. Usually there is positivity for antineutrophil cytoplasmatic antigene (ANCA) but its absence does not exclude this diagnosis, especially when the clinical picture is highly suggestive $[4,5]$.

We present a case of palsy of the lower cranial nerves as the initial presentation of a seronegative WG.

\section{Case Report}

A 59 years old woman was in her usual state of good health until October 2009. She presented in the emergency room with one month history of severe right otalgy followed by an indolent and progressive appearance of homolateral facial palsy, gait disturbance, dysphagia and dysphonia. There was low grade fever since the beginning, without other accompanying symptoms. She had previously been treated with antibiotic (ceftriaxone $1 \mathrm{~g} \mathrm{IM}$ for 2 weeks) and oral steroid (deflazacort up to $30 \mathrm{mg}$ PO for 7 days) with no recovery. On the neurological examination we found peripheral type paresis of the VII, IX, X and $\mathrm{XI}^{\text {th }}$ right cranial nerves, and the rest of the examination was unremarkable. Also, there were no significant signs in the general examination namely adenopathies or cutaneous abnormalities.

Since there was a paresis of the lower cranial nerves, the syndromic diagnosis of Vernet Syndrome was done, and a lesion in the jugular foramen was suspected. A brain MRI was performed and found out a round mass in the expected location, with homogeneous enhancement after the use of gadolinium, and also a right mastoiditis. The MRA supported a possible jugular glomus (Figure 1). In this context, a conventional angiography was realized and the hypothesis of glomus was not confirmed, but an occlusion of the right internal jugular vein was identified. The presumptive diagnosis a jugular vein thrombosis due to a right otomastoiditis was assumed. However, taking into account the age of the patient, further investigation was made to exclude prothrombotic states or occult neoplasy.

The laboratory evaluation disclosed a modest elevation of ESR (45 $\mathrm{mm}$ ) and CRP (3.07 mg/dl), leucocytosis without neutrophilia (11,700/ $\mu \mathrm{l})$ and an urinalysis showed mild microhematuria $(52.8 / \mu \mathrm{l})$. The prothrombotic screening which includes an auto-immune evaluation was completely normal (including ANA and ANCA). Renal US and CT scan did not find any anatomic abnormalities or signs of medical

*Corresponding author: Sara Machado, Neurology Department, Hospital Professor Doutor Fernando Fonseca, EPE, IC-19 Amadora, Portugal, Tel: +351 214348404; E-mail: s.jose.11@ucl.ac.uk

Received February 18, 2014; Accepted March 12, 2014; Published March 15 2014

Citation: Machado S, Inácio N, Paulas F, Biscoito L, Farias JP, et al. (2014) Seronegative Wegener's Granulomatosis Presenting as a Multiple Cranial Neuropathy. J Neurol Disord 2: 149. doi:10.4172/2329-6895.1000149

Copyright: (c) 2014 Machado S, et al. This is an open-access article distributed under the terms of the Creative Commons Attribution License, which permits unrestricted use, distribution, and reproduction in any medium, provided the original author and source are credited. 


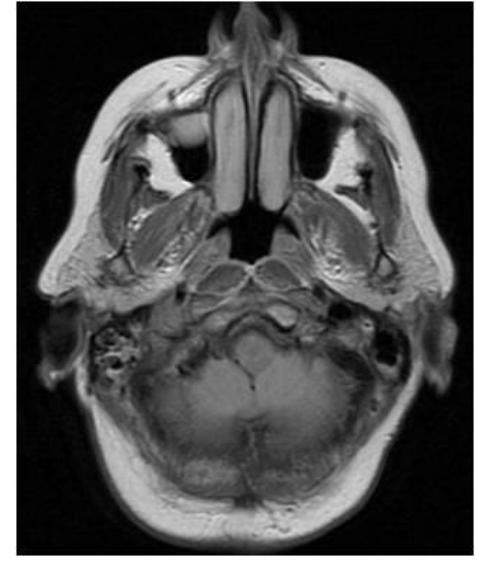

Figure 1: Tissular formation in the right jugular foramen.

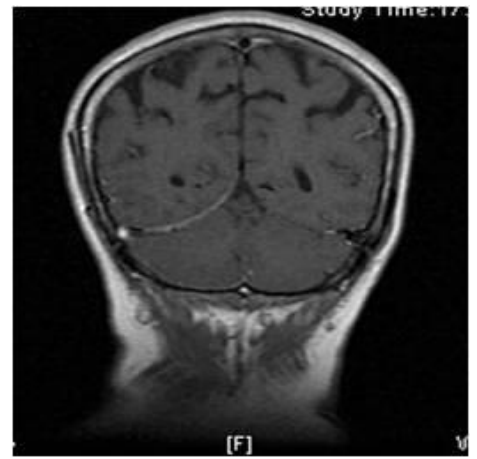

Figure 2: Duramater enhancement after gadolinium administration.

nephropathy. A thoracic CT scan found multiple millimetric bilateral pulmonary nodules, interpreted as metastasis. A comprehensive search for a primary lesion was performed with several exams but without any positive results: tumoral markers, mammography and mammary US and PET scan. CSF analysis including cytology, bacterial and viral antibody detection (such as antibodies against varicella zoster and herpes simplex I and II virus and against mycoplasma and borrelia burgdorferi), immune screening (including ANA, ANCA and ECA), oligoclonal bands and cultures (namely for mycobacterium tuberculosis) were normal.

During the hospital stay there was a spontaneous but subtle improvement. She was discharged with acetylsalicylic acid $150 \mathrm{mg}$ $\mathrm{PO}$ and then seen in regular visits in both Neurology and Oncology outpatient clinics. In April 2010, she returned to our department with a week course of severe headache which was refractory to NSAIDs and difficulty in the verbal articulation de novo. On the examination there was only a XII ${ }^{\text {th }}$ right cranial nerve palsy with no meningeal signs or other remarkable deficits. A MRI was performed and the previous right mastoiditis and ipsilateral mass in the jugular foramen both persisted and a new adjacent meningeal enhancement was found (Figure 2). A second lumbar puncture was done, with a normal opening pressure, but this time with pleocytosis ( 42 cells, mononuclear). Apart from these finding, the LCR analysis was normal once again (with all the evaluations previously described).

Based on the upper and inferior airways, renal and CNS involvement plus the absence of any abnormal values of immune marker as ANCA, the diagnosis of seronegative granulomatous vasculitis was likely and the patient was referred to the systemic immunomediated diseases outpatient clinic. Corticotherapy was then initiated with three IV pulses of methylprednisolone followed by a month course of prednisolone 1 $\mathrm{mg} / \mathrm{kg}$ PO. All the clinical symptoms disappeared, a MRI was repeated with no meningeal enhancement, and the LCR evaluation was entirely normal. As this case represented a disseminated form of Wegener's Granulomatosis, we initiated the treatment with cyclophosphamide. There were administrated 7 cycles, 3 of $500 \mathrm{mg}$ and 4 of $450 \mathrm{mg}$ IV, with an exceptional clinical recovery. The control MRI showed a decrease of the primary lesion and no enhancement after gadolinium (Figure 3). The controle chest CT scan found only 2 of the previous micronodules and both the urinary sediment and the ER normalized. After the suspension of this drug, azathyoprine was started but due to gastrointestinal intolerance, mycophenolate mophetil was finally initiated. She is currently taking $500 \mathrm{mg}$ PO tid. After 13 months of treatment she remains completely asymptomatic without any abnormalities in the neurological examination.

\section{Discussion}

WG is a multisystemic granulomatous disease that usually begins with a localized inflammation of the nasal mucosa, lung tissue and kidneys and may subsequently involve other systems as the nervous system. The presence of cranial nerves involvement is not common but can develop in $6.5 \%$ of cases [5]. To our knowledge, cranial nerve palsies without previously diagnosis of WG is very rarely seen in the literature and lower cranial nerve palsies when present, may be associated with a skull-base pachymeningitis [6,7]. The aim of this report is to draw physician's attention to the high possibility of unusual presentations of WG.

This entity should thus be considered in cranial multiple neuropathies or aseptic meningitis when there is also renal and respiratory tract involvement. In our case, these clues were all present: cranial neuropathy and inflammatory changes in the CSF not explained by other inflammatory disorders (namely sarcoid) or infections (such as borreliosis), as well as the renal and pulmonary abnormalities.

Although ANCA positivity is a sensitive and specific marker or WG, it is not currently part of the disease classification and the seronegativity should not exclude this diagnosis and delay the prompt initiation of treatment. This patient had symptomatic improvement after treatment with prednisolone and cyclophospamide.

It's early diagnosis and treatment is of paramount importance

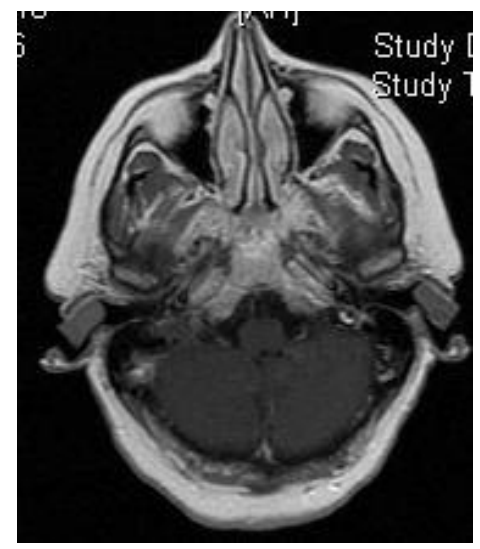

Figure 3: Size reduction of the tissular formation in the right jugular foramen and absence of meningeal enhancement after gadolinium administration. 
Citation: Machado S, Inácio N, Paulas F, Biscoito L, Farias JP, et al. (2014) Seronegative Wegener's Granulomatosis Presenting as a Multiple Cranial Neuropathy. J Neurol Disord 2: 149. doi:10.4172/2329-6895.1000149

as it is well known that without therapy it is uniformly fatal, being its 1 -year mortality $80 \%$. Corticosteroids and cytotoxic agents as cyclophosphamide are the mainstay of treatment and have significantly improved the natural course of the disease [8,9]. Prompt recognition and immunosuppressive therapy can have a major impact on the ominous course of the disease.

\section{References}

1. Holle JU, Gross WL (2011) Neurological involvement in Wegener's granulomatosis. Curr Opin Rheumatol 23: 7-11.

2. Kohlberg GD, Truong MT, Chang KW (2010) Wegener's granulomatosis in an adolescent presenting with pachymeningitis, mastoid effusion and Horner's syndrome. International Journal of Pediatric Ororhinolaryngology Extra 6: 314.

3. Leavitt RY, Fauci AS, Bloch DA, Michel BA, Hunder GG, et al. (1990) The American College of Rheumatology 1990 criteria for the classification of Wegener's Granulomatosis. Arthritis Rheum 33: 1101-1107.

4. Yildirim N, Arslanoglu A, Aygun N (2008) Otologic and leptomeningeal involvements as presenting features in seronegative Wegener granulomatosis. Am J Otolaryngol 29: 147-149.

5. Reinhold-Keller E, de Groot K, Holl-Ulrich K, Arlt AC, Heller M, et al. (2001) Severe CNS manifestations as the clinical hallmark in generalized Wegener's granulomatosis consistently negative for antineutrophil cytoplasmic antibodies (ANCA). A report of 3 cases and a review of the literature. Clin Exp Rheumato 19: 541-549.

6. Reza MJ, Dornfeld L, Goldberg LS, Bluestone R, Pearson CM (1975) Wegener's granulomatosis. Long-term followup of patients treated with cyclophosphamide. Arthritis Rheum 18: 501-506.

7. Iqbal AM, Blackburn D, Rafiq M, Sharrack B (2013) Wegener's granulomatosis presenting with multiple cranial nerve palsies and pachymeningitis. Pract Neurol 13: 193-195.

8. Nishino H, Rubino FA, DeRemee RA, Swanson JW, Parisi JE (1993) Neurological involvement in Wegener's granulomatosis: an analysis of 324 consecutive patients at the Mayo Clinic. Ann Neurol 33: 4-9.

9. Fauci AS, Haynes BF, Katz P, Wolff SM (1983) Wegener's granulomatosis: prospective clinical and therapeutic experience with 85 patients for 21 years. Ann Intern Med 98: 76-85. 